hep-th/0509016

SWAT-05-439

\title{
MHV-Vertices for Gravity Amplitudes
}

\author{
N. E. J. Bjerrum-Bohr ${ }^{1}$, David C. Dunbar ${ }^{1}$, Harald Ita ${ }^{1}$, Warren B. Perkins ${ }^{1}$ \\ and Kasper Risager ${ }^{2}$ \\ ${ }^{1}$ Department of Physics, University of Wales Swansea, \\ Swansea, SA2 8PP, UK \\ ${ }^{2}$ Niels Bohr Institute, Blegdamsvej 17, DK-2100, Copenhagen, Denmark
}

\begin{abstract}
We obtain a CSW-style formalism for calculating graviton scattering amplitudes and prove its validity through the use of a special type of BCFW-like parameter shift. The procedure is illustrated with explicit examples.

KEYwords: Models of Quantum Gravity, Supergravity Models.
\end{abstract}




\section{Contents}

1. Introduction 1

2. Graviton Scattering Amplitudes 3

3. NMHV Graviton Scattering Amplitudes 5

3.1 Five-Point Example $M\left(1^{-}, 2^{-}, 3^{-}, 4^{+}, 5^{+}\right)$

3.2 Remarks on the Twistor Space Structure of MHV Gravity Amplitudes 9

4. Beyond NMHV 10

4.1 General CSW rules 10

4.2 Example 11

4.3 Proof of MHV-vertex rules 13

4.4 MHV-vertex Expansion for $\mathrm{N}^{2} \mathrm{MHV}$ Amplitudes 13

4.5 General Case 15

5. Conclusions and Comments 16

\section{Introduction}

In a helicity formalism the simplest Yang-Mills amplitudes are the MHV amplitudes where precisely two external gluons have negative helicity and the remaining legs all have positive helicity. If legs $j$ and $k$ have negative helicity, the colour-ordered [1] partial amplitude takes the form [2],

$$
A_{n}^{\text {tree }}\left(1^{+}, \ldots, j^{-}, \ldots, k^{-}, \ldots, n^{+}\right)=i \frac{\langle j k\rangle^{4}}{\langle 12\rangle\langle 23\rangle \cdots\langle n 1\rangle} .
$$

We use the notation $\langle j l\rangle \equiv\left\langle j^{-} \mid l^{+}\right\rangle,[j l] \equiv\left\langle j^{+} \mid l^{-}\right\rangle$, with $\left|i^{ \pm}\right\rangle$being massless Weyl spinors with momentum $k_{i}$ and chirality $\pm[3,4]$. The spinor products are related to momentum invariants by $\langle i j\rangle[j i]=2 k_{i} \cdot k_{j} \equiv s_{i j}$ with $\langle i j\rangle^{*}=[j i]$. As in twistor-space studies we define,

$$
\lambda_{i}=\left|i^{+}\right\rangle, \quad \bar{\lambda}_{i}=\left|i^{-}\right\rangle .
$$

Inspired by the duality between twistor string theory and Yang-Mills [5] (and generalising a previous description of the simplest gauge theory amplitudes by Nair [6]), Cachazo, Svrček and Witten proposed a reformulation of perturbation theory in terms of off-shell MHV-vertices [7], which can be depicted, 


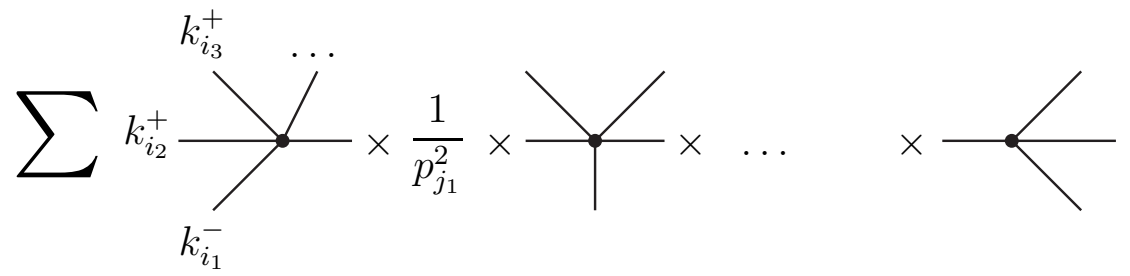

The off-shell continuation for a leg of momentum $p$ was achieved by replacing $\lambda(p)$ by,

$$
\lambda_{a}(p)=p_{a \dot{a}} \bar{\eta}^{\dot{a}},
$$

where $\bar{\eta}^{\dot{a}}$ is an arbitrary reference spinor. While individual CSW diagrams depend on $\bar{\eta}$, the full amplitude is $\bar{\eta}$-independent. This reformulation has led to or inspired a variety of calculational advances, both for tree level scattering [8] and at loop level [9] in Yang-Mills theory.

This reformulation has been demonstrated to reproduce all known results for gluon scattering at tree level and, often, gives relatively simple expressions for these amplitudes. Although originally given for gluon scattering only, these rules have been shown to extend to other types of massless particle [10] and indeed to massive particles [11]. It has been shown [12-14] that, with the correct off-shell prescription, these vertices can be used to reproduce known one-loop results $[15,16]$ in supersymmetric theories.

In an alternate approach to computing tree level amplitudes, Britto, Cachazo, Feng and Witten [17] obtained a recursion relation based on analytically shifting a pair of external legs,

$$
\lambda_{i} \longrightarrow \lambda_{i}+z \lambda_{j}, \quad \bar{\lambda}_{j} \longrightarrow \bar{\lambda}_{j}-z \bar{\lambda}_{i},
$$

and determining the physical amplitude, $A_{n}(0)$, from the poles in the shifted amplitude, $A_{n}(z)$. This leads to a recursion relation in the number of external legs, $n$, of the form,

$$
A_{n}(0)=\sum_{\alpha} \hat{A}_{n-k_{\alpha}+2}\left(z_{\alpha}\right) \times \frac{i}{P_{\alpha}^{2}} \times \hat{A}_{k_{\alpha}}\left(z_{\alpha}\right),
$$

where the factorisation is only on these poles, $z_{\alpha}$, where legs $i$ and $j$ are connected to different sub-amplitudes. This is depicted below:

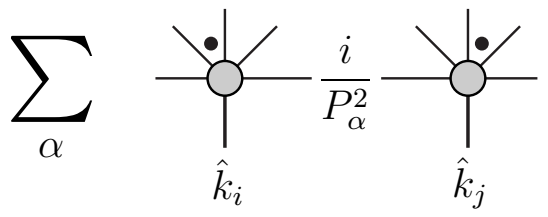


These recursion relations also give relatively compact formulae for tree amplitudes $[18,19]$. Recursion relations based on analyticity can also be used at loop level both to calculate rational terms [20] and the coefficients of integral functions [21].

The factorisation properties of the amplitudes seem to lie at the heart of both approaches. In both cases the amplitude is expressed as a sum of its factorisations in a well specified manner. As such, one might hope to derive the MHV-vertex formulation by applying an analytic shift and obtaining a recursion relation. In ref. [22] it was demonstrated that such shifts exist and can be used to derive the MHV vertex approach in gauge theory. The shift affects all of the negative helicity legs, $k_{m_{i}}$,

$$
\bar{\lambda}_{m_{i}} \rightarrow \hat{\bar{\lambda}}_{m_{i}}=\bar{\lambda}_{m_{i}}+z r_{i} \bar{\eta}
$$

with the $r_{i}$ chosen to ensure momentum conservation.

Most of the above developments have been made for gauge theory amplitudes. The existence of a BCFW recusion relation for gravity amplitudes was strongly supported in [24,25], and in this article we construct a CSW approach using the newly established shift (1.6) under the assumption that gravity amplitudes are sufficiently well behaved for large values of $z$ in (1.6).

The key ingredient in obtaining the MHV rules is the analytic structure of the amplitude which also underlies the derivation of the recursion relations. In this context it becomes clear that these two formalisms have their roots in the same physical behaviour of on-shell amplitudes.

\section{Graviton Scattering Amplitudes}

Graviton scattering amplitudes are generally considerably more complicated than those for gauge theory. To date, explicit expressions have only been given for the MHV amplitudes [23, 24] and for the six-point NMHV amplitude [25, 26]. (As for gauge theories, amplitudes with all helicities identical vanish, as do those with one different, $M\left(1^{ \pm}, 2^{+}, 3^{+}, \cdots, n^{+}\right)=0$.)

In principle, gravity amplitudes can be constructed through the Kawai, Lewellen and Tye (KLT)-relations [27]. The explicit forms of these, up to six points, are,

$$
\begin{aligned}
M_{3}^{\text {tree }}(1,2,3)= & -i A_{3}^{\text {tree }}(1,2,3) A_{3}^{\text {tree }}(1,2,3), \\
M_{4}^{\text {tree }}(1,2,3,4)= & -i s_{12} A_{4}^{\text {tree }}(1,2,3,4) A_{4}^{\text {tree }}(1,2,4,3), \\
M_{5}^{\text {tree }}(1,2,3,4,5)= & i s_{12} s_{34} A_{5}^{\text {tree }}(1,2,3,4,5) A_{5}^{\text {tree }}(2,1,4,3,5) \\
& +i s_{13} s_{24} A_{5}^{\text {tree }}(1,3,2,4,5) A_{5}^{\text {tree }}(3,1,4,2,5), \\
M_{6}^{\text {tree }}(1,2,3,4,5,6)= & -i s_{12} s_{45} A_{6}^{\text {tree }}(1,2,3,4,5,6)\left(s_{35} A_{6}^{\text {tree }}(2,1,5,3,4,6)\right. \\
& \left.+\left(s_{34}+s_{35}\right) A_{6}^{\text {tree }}(2,1,5,4,3,6)\right)+\mathcal{P}(2,3,4),
\end{aligned}
$$


where $\mathcal{P}(2,3,4)$ represents the sum over permutations of legs $2,3,4$ and the $A_{n}^{\text {tree }}$ are the tree-level colour-ordered gauge theory partial amplitudes. We have suppressed factors of $g^{n-2}$ in the $A_{n}^{\text {tree }}$, as well as a factor of $(\kappa / 2)^{n-2}$ in the gravity amplitude.

This formulation allows results from Yang-Mills theory to be recycled in theories of gravity and supergravity [28-32]. While these relations are directly applicable to tree amplitudes, this formulation also has implications for loop amplitude calculations, particularly in unitarity based methods where the tree amplitudes are used to compute the loop amplitudes [33-35]. Consequently, similar relationships can hold for the coefficients of integral functions [36].

Although, in principle, the KLT relations can be used to calculate gravity tree amplitudes, they have several undesirable features. Firstly, the factorisation structure is rather obtuse. The Yang-Mills tree amplitudes contain single poles so we might expect un-physical double poles to appear in the sum. These are actually canceled by the multiplying momentum factors, but often in a non-trivial manner. Secondly, the expressions do not tend to be compact as the permutation sums grow rather quickly with the number of points. In fact, the Berends, Giele and Kuijf (BGK) form of the MHV gravity amplitude [23],

$$
\begin{array}{r}
M_{n}^{\text {tree }}\left(1^{-}, 2^{-}, 3^{+}, \cdots, n^{+}\right) \\
=-i\langle 12\rangle^{8} \times\left[\frac{[12][n-2 n-1]}{\langle 1 n-1\rangle N(n)}\left(\prod_{i=1}^{n-3} \prod_{j=i+2}^{n-1}\langle i j\rangle\right) \prod_{l=3}^{n-3}\left(-\left[n\left|K_{l+1, n-1}\right| l\right\rangle\right)\right. \\
+\mathcal{P}(2,3, \cdots, n-2)]
\end{array}
$$

is rather more compact than that of the KLT sum (as is the expression in [24].) In the above we use the definitions,

$$
\left[k\left|K_{i, j}\right| l\right\rangle \equiv\left\langle k^{+}\left|K_{i, j}\right| l^{+}\right\rangle \equiv\left\langle l^{-}\left|K_{i, j}\right| k^{-}\right\rangle \equiv\left\langle l\left|K_{i, j}\right| k\right] \equiv \sum_{a=i}^{j}[k a]\langle a l\rangle,
$$

and $N(n)=\prod_{1 \leq i<j \leq n}\langle i j\rangle$.

Both the KLT form of the MHV amplitude (2.1) and the above form (2.2) display a feature not shared by the Yang-Mills expressions: they not only depend on the holomorphic variables $\lambda$, but also on the $\bar{\lambda}$ - within the $s_{i j}$ for the KLT expression and explicitly in the BGK expression. In both cases this dependence is polynomial in the numerator. This feature complicates the twistor space structure of any potential form of a MHV vertex for gravity. For Yang-Mills, the holomorphic vertex corresponds simply to points lying on a line in twistor space. For gravity the picture will be of points lying on the "derivative of a $\delta$-function" [5]. The practical difference is that both $\lambda(q)$ and $\bar{\lambda}(q)$ must be correctly continued off-shell. (The exception to this is the three-point vertex for which the gravity MHV expression is holomorphic.) 
Various attempts have been made to find the off-shell continuation [37,38]. Despite the failure to find a MHV vertex formulation, gravity amplitudes are amenable to recursive techniques [24,25]. In [39] a current algebra formulation was demonstrated for the MHV gravity amplitudes which also suggests that a MHV vertex might exist.

\section{NMHV Graviton Scattering Amplitudes}

We shall demonstrate the off-shell MHV vertex for gravity using the analytic structure of the amplitudes with three negative helicity legs (known as "next-to-MHV" or NMHV amplitudes). The shift of [22] allows us to rewrite the NMHV amplitudes as products of MHV-amplitudes and thus gives a CSW type expansion for these amplitudes directly, from which we can identify the off-shell gravity MHV-vertices.

Let us start by considering a generic $n$-point NMHV graviton amplitude $M_{n}\left(m_{1}^{-}\right.$, $m_{2}^{-}, m_{3}^{-}, \cdots, n^{+}$), where we label the three negative helicity legs 1,2 and 3 by $m_{i}$. We can make the same continuation as in the Yang-Mills case,

$$
\begin{aligned}
& \hat{\bar{\lambda}}_{m_{1}}=\bar{\lambda}_{m_{1}}+z\left\langle m_{2} m_{3}\right\rangle \bar{\eta}, \\
& \hat{\bar{\lambda}}_{m_{2}}=\bar{\lambda}_{m_{2}}+z\left\langle m_{3} m_{1}\right\rangle \bar{\eta}, \\
& \hat{\bar{\lambda}}_{m_{3}}=\bar{\lambda}_{m_{3}}+z\left\langle m_{1} m_{2}\right\rangle \bar{\eta},
\end{aligned}
$$

which shifts the momentum of the negative helicity legs,

$$
\hat{k}_{m_{i}}(z)=\lambda_{m_{i}}\left(\bar{\lambda}_{m_{i}}+z\left\langle m_{i-1} m_{i+1}\right\rangle \bar{\eta}\right),
$$

but leaves them on-shell, $k_{m_{i}}(z)^{2}=0$, while the combination $k_{m_{1}}(z)+k_{m_{2}}(z)+k_{m_{3}}(z)$ is independent of $z$. Under the shift we obtain the analytic continuation of the amplitude $M_{n}(z)=\hat{M}_{n}$ into the complex plane. We use a 'hat' to distinguish the unshifted objects, $a$, from the shifted ones, $\hat{a}$.

For a shifted amplitude we can evaluate the following contour integral at infinity,

$$
\frac{1}{2 \pi i} \oint \frac{d z}{z} M_{n}(z)=C_{\infty}=M_{n}(0)+\sum_{\alpha} \operatorname{Res}_{z=z_{\alpha}} \frac{M_{n}(z)}{z} .
$$

If $M_{n}(z)$ is rational with simple poles at points $z_{\alpha}$ and $C_{\infty}$ vanishes, $M_{n}(0)$ can be expressed in terms of residues,

$$
M_{n}(0)=-\sum_{\alpha} \operatorname{Res}_{z=z_{\alpha}} \frac{M_{n}(z)}{z} .
$$

The first condition is satisfied as a result of the general factorisation properties of amplitudes, however the second is difficult to prove in general for gravity amplitudes.

The shifted amplitude has poles in $z$ whenever a momentum invariant $\hat{P}^{2}(z)$ vanishes. Given the form of the shift, all momentum invariants apart from those 
containing all or none of the negative helicities are $z$-dependent. Thus the NMHV amplitudes have factorisations where two of the negative helicity legs lie on one side and one on the other. For the above shift it can be checked that all factorisations involving the MHV googly 3-point amplitude $M(-++)$ vanish. All poles of the amplitude must therefore factorise as,

$$
M^{\mathrm{MHV}}\left(m_{i_{1}}^{-}, \cdots, P^{-}\right) \times \frac{i}{P^{2}} \times M^{\mathrm{MHV}}\left((-P)^{+}, m_{i_{2}}^{-}, m_{i_{3}}^{-}, \cdots\right)
$$

for $i_{k}=1,2$ or 3 , as we expect for a CSW-type expansion. $\hat{P}^{2}(z)$ vanishes linearly in $z$, so $M_{n}(z)$ has simple poles when $z_{\alpha}$ satisfies,

$$
\hat{P}^{2}=P^{2}+z_{\alpha}\left\langle m_{i_{2}} m_{i_{3}}\right\rangle\left[\eta|P| m_{i_{1}}\right\rangle=0 .
$$

The residue at each pole is just the product of the two MHV tree amplitudes evaluated at $z=z_{\alpha}$. Spinor products $\langle i j\rangle$ which are not $z_{\alpha}$ dependent take their normal values, while terms like $\langle i \hat{P}\rangle$ are evaluated by noting,

$$
\langle i \hat{P}\rangle=\frac{\langle i \hat{P}\rangle[\hat{P} \eta]}{[\hat{P} \eta]}=\frac{\langle i|P| \eta]}{\omega}
$$

where $P$ is the unshifted form. The objects $\omega$ will cancel between the two tree amplitudes since the product has zero spinor weight in $P$. This substitution is precisely the CSW prescription, $\lambda(P) \longrightarrow P \mid \eta$.

For Yang-Mills this would be all we need, but for gravity we must also consider substitutions for $[i j]$ where $i$ and/or $j$ are one of the negative helicities or $\hat{P}$. These substitutions are of the form,

$$
\begin{aligned}
{\left[l^{+} \hat{P}\right] } & =\frac{\left[l^{+} \hat{P}\right]\langle\hat{P} \alpha\rangle}{\langle\hat{P} \alpha\rangle}=\frac{\omega\left[l^{+}|\hat{P}| \alpha\right\rangle}{[\eta|P| \alpha\rangle}=\frac{\omega\left[l^{+}|P| m_{i_{1}}\right\rangle}{\left[\eta|P| m_{i_{1}}\right\rangle}, \\
{\left[\hat{m}_{i_{2}} \hat{m}_{i_{3}}\right] } & =\left[m_{i_{2}} m_{i_{3}}\right]+z_{\alpha}\left[\eta\left|P_{m_{i_{2}} m_{i_{3}}}\right| m_{i_{1}}\right\rangle \\
{\left[\hat{m}_{i_{1}} l^{+}\right] } & =\left[m_{i_{1}} l^{+}\right]+z_{\alpha}\left[\eta l^{+}\right]\left\langle m_{i_{2}} m_{i_{3}}\right\rangle
\end{aligned}
$$

where $l^{+}$denotes a positive helicity leg. We choose the arbitrary spinor $\alpha$ to be $m_{i_{1}}$ in order to replace $\hat{P}$ by $P$. Equations (3.7) and (3.8) are the specific substitutions that determine the value of the MHV amplitudes on the pole and thus the MHV vertices.

Note that the form of the off-shell continuation,

$$
\hat{\bar{\lambda}}_{m_{1}}=\bar{\lambda}_{m_{1}}+z\left\langle m_{2} m_{3}\right\rangle \bar{\eta}=\bar{\lambda}_{m_{1}}-\frac{P^{2} \bar{\eta}}{\left[\eta|P| m_{1}\right\rangle},
$$

can be interpreted as yielding contact terms since the $P^{2}$ factor may cancel the pole. 
We conclude that the NMHV graviton scattering amplitude can be expressed in terms of MHV vertices as,

$$
\begin{aligned}
M_{n}\left(1^{-}, 2^{-}, 3^{-}, 4^{+}, \ldots, n^{+}\right)= & \sum_{r=0}^{n-4} \sum_{\mathcal{P}\left(i_{1}, i_{2}, i_{3}\right)} \sum_{\mathcal{P}\left(d_{i}\right)} M_{r+3}^{\mathrm{MHV}}\left(\hat{m}_{i_{2}}^{-}, \hat{m}_{i_{3}}^{-}, d_{1}^{+}, \cdots, d_{r}^{+}, \hat{P}^{+}\right)\left(z_{r}\right) \\
& \times \frac{i}{P_{m_{i_{1}} d_{r+1} \cdots, n}^{2}} \times M_{n-r-1}^{\mathrm{MHV}}\left((-\hat{P})^{-}, \hat{m}_{i_{1}}^{-}, d_{r+1}^{+}, \cdots, n\right)\left(z_{r}\right) .
\end{aligned}
$$

Here the sums over $\mathcal{P}_{r}\left(i_{1}, i_{2}, i_{3}\right)$ and $\mathcal{P}_{r}\left(d_{i}\right)$ are respectively sums over those permutations of the negative and positive helicity legs that swap legs between the two MHV vertices.

We now turn to the discussion of the behaviour of $M_{n}(z)$ for large $z$. By naive power counting one might expect shifted gravity amplitudes to diverge at large $z$. However in both ref. [24] and ref. [25] it was established by various techniques, including numerical studies, that NMHV gravity amplitudes do vanish asymptotically under the BCFW shift. This behaviour is difficult to prove either by analysing Feynman diagrams or using the KLT relations, since large cancellations are inherent in both formalisms. Under the shift (3.1) the amplitudes we have examined are very well behaved at large $z$, with

$$
M_{6,7}(z) \sim \frac{1}{z^{5}}
$$

for both the six and seven point NMHV amplitudes. This is a much stronger behaviour than under the BCF shift where, $M_{6,7}(z) \sim \frac{1}{z}$.

If we choose a specific value for the reference spinor,

$$
\bar{\eta}=\bar{\lambda}_{a}
$$

where $a$ is one of the positive helicity legs, then the shift we use is a combination of three BCF shifts involving the three negative helicity legs and a positive helicity leg $a$

$$
\begin{array}{ll}
\lambda_{a} \longrightarrow \lambda_{a}+z_{1}\langle 23\rangle \lambda_{1}, & \bar{\lambda}_{1} \longrightarrow \bar{\lambda}_{1}-z_{1}\langle 23\rangle \bar{\lambda}_{a}, \\
\lambda_{a} \longrightarrow \lambda_{a}+z_{2}\langle 31\rangle \lambda_{2}, & \bar{\lambda}_{2} \longrightarrow \bar{\lambda}_{2}-z_{2}\langle 31\rangle \bar{\lambda}_{a}, \\
\lambda_{a} \longrightarrow \lambda_{a}+z_{3}\langle 12\rangle \lambda_{3}, & \bar{\lambda}_{3} \longrightarrow \bar{\lambda}_{3}-z_{3}\langle 12\rangle \bar{\lambda}_{a},
\end{array}
$$

with $z_{1}=z_{2}=z_{3}$. The shift on $\lambda_{a}$ vanishes due to the Schouten identity. In ref. [25] it was proven that the amplitude vanishes at infinity under a single shift of this form, providing further evidence that the NMHV amplitude vanishes asymptotically under the shift (3.1).

\subsection{Five-Point Example $M\left(1^{-}, 2^{-}, 3^{-}, 4^{+}, 5^{+}\right)$}

In this section we show, using an explicit example, how MHV vertices can be assembled into graviton scattering amplitudes. The first non-trivial example is the 
five-point amplitude, $M\left(1^{-}, 2^{-}, 3^{-}, 4^{+}, 5^{+}\right)$. This is a 'googly' amplitude as it can be obtained by conjugating the five-point MHV amplitude. As above we shift the negative helicity legs, here $k_{1}, k_{2}$ and $k_{3}$, and compute the residues of the amplitude $M\left(\hat{1}^{-}, \hat{2}^{-}, \hat{3}^{-}, 4^{+}, 5^{+}\right)(z)$. The expansion in terms of MHV vertices is non-trivial and reveals the structure of the MHV vertices.

Up to relabeling we have two types of residue,

$$
\begin{aligned}
& D_{1}\left(1^{-}, 2^{-}, 3^{-}, 4^{+}, 5^{+}\right)=M\left(\hat{2}^{-}, \hat{3}^{-}, \hat{p}^{+}\right) \times \frac{i}{s_{23}} \times M\left((-\hat{p})^{-}, 4^{+}, 5^{+}, \hat{1}^{-}\right), \\
& D_{2}\left(1^{-}, 2^{-}, 3^{-}, 4^{+}, 5^{+}\right)=M\left(\hat{2}^{-}, \hat{3}^{-}, 4^{+}, \hat{p}^{-}\right) \times \frac{i}{s_{15}} \times M\left((-\hat{p})^{+}, 5^{+}, \hat{1}^{-}\right),
\end{aligned}
$$

which can be associated to the CSW diagrams,
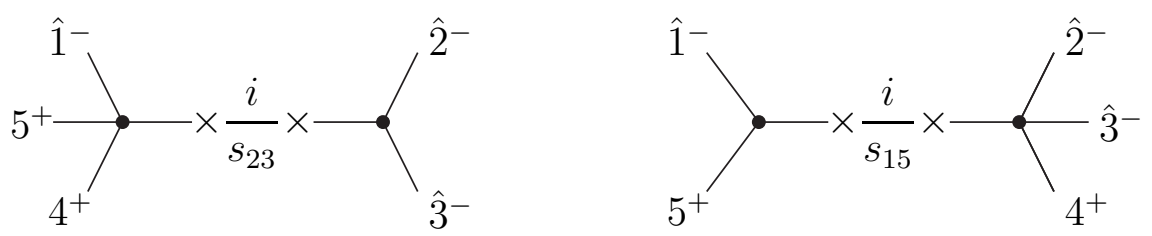

Explicitly we find for the three-point function,

$$
M\left(\hat{2}^{-}, \hat{3}^{-}, \hat{p}^{+}\right)=i \frac{\langle 23\rangle^{6}}{\langle 2 \hat{p}\rangle^{2}\langle\hat{p} 3\rangle^{2}}=i \frac{\omega^{4}\langle 23\rangle^{6}}{\left[\eta\left|P_{23}\right| 2\right\rangle^{2}\left[\eta\left|P_{23}\right| 3\right\rangle^{2}} .
$$

The four point amplitude can be expressed in several ways, including,

$$
\begin{aligned}
M_{4}\left((-\hat{p})^{-}, 4^{+}, 5^{+}, \hat{1}^{-}\right) & =-i s_{45} A_{4}\left((-\hat{p})^{-}, 4^{+}, 5^{+}, \hat{1}^{-}\right) A\left((-\hat{p})^{-}, 5^{+}, 4^{+}, \hat{1}^{-}\right) \\
& =\frac{i s_{45}\langle\hat{p} 1\rangle^{8}}{\langle\hat{p} 4\rangle\langle 45\rangle\langle 51\rangle\langle 1 \hat{p}\rangle\langle\hat{p} 5\rangle\langle 54\rangle\langle 41\rangle\langle 1 \hat{p}\rangle} \\
& =\frac{i[45]}{\langle 14\rangle\langle 15\rangle\langle 45\rangle} \frac{\omega^{-4}\left[\eta\left|P_{23}\right| 1\right\rangle^{6}}{\left[\eta\left|P_{23}\right| 4\right\rangle\left[\eta\left|P_{23}\right| 5\right\rangle}
\end{aligned}
$$

giving the tree diagram as,

$$
D_{1}\left(1^{-}, 2^{-}, 3^{-}, 4^{+}, 5^{+}\right)=\frac{i[45]\left[\eta\left|P_{23}\right| 1\right\rangle^{6}}{\langle 14\rangle\langle 15\rangle\langle 45\rangle\left[\eta\left|P_{23}\right| 4\right\rangle\left[\eta\left|P_{23}\right| 5\right\rangle} \frac{i}{s_{23}} \frac{i\langle 23\rangle^{6}}{\left[\eta\left|P_{23}\right| 2\right\rangle^{2}\left[\eta\left|P_{23}\right| 3\right\rangle^{2}} .
$$

For this particular diagram the prescription implied by the shift is equivalent to the CSW rules for gauge theory as there is no need to find a continuation for $\bar{\lambda}$.

For $D_{2}$ we find,

$$
D_{2}\left(1^{-}, 2^{-}, 3^{-}, 4^{+}, 5^{+}\right)=\frac{i\langle 23\rangle^{7}[\hat{2} \hat{3}]}{\langle 24\rangle\langle 34\rangle\left[\eta\left|P_{15}\right| 4\right\rangle^{2}\left[\eta\left|P_{15}\right| 2\right\rangle\left[\eta\left|P_{15}\right| 3\right\rangle} \frac{i}{s_{15}} \frac{i\left[\eta\left|P_{15}\right| 5\right\rangle^{6}}{\left[\eta\left|P_{15}\right| 1\right\rangle^{2}\langle 51\rangle^{2}} .
$$


This differs from the simple CSW prescription in the definition of [ $[\hat{2} \hat{3}]$. Here,

$$
\begin{gathered}
{[\hat{2} \hat{3}]=[23]+z(\langle 31\rangle[\eta 3]+\langle 12\rangle[2 \eta])=[23]+z\left[\eta\left|P_{23}\right| 1\right\rangle} \\
=[23]-\frac{P_{15}^{2}}{\langle 23\rangle\left[\eta\left|P_{15}\right| 1\right\rangle}\left[\eta\left|P_{23}\right| 1\right\rangle .
\end{gathered}
$$

With this substitution we can verify that the sum of diagrams is independent of $\bar{\eta}$ and equal to the conjugate of the five-point MHV tree amplitude.

For the six-point amplitude there are three diagrams. We have explicitly checked that the sum over permutations of these diagrams is equal to the known form of the six-point NMHV amplitude $[25,26]$. Seven point NMHV amplitudes can be obtained explicitly using the KLT relationships - at least using computer algebra. We have checked numerically that the seven-point amplitudes obtained from the MHV vertices match those obtained from the KLT relation.

\subsection{Remarks on the Twistor Space Structure of MHV Gravity Amplitudes}

The twistor space structure of an amplitude refers to the support of the amplitude after it has been Penrose transformed into twistor space variables. As was shown in ref. [5], the twistor space support of an amplitude can be tested by simply acting with certain differential operators, without having to resort to Penrose or Fourier transformations. The operator of particular interest is the 'collinearity operator',

$$
\left[F_{i j k}, \eta\right]=\langle i j\rangle\left[\frac{\partial}{\partial \bar{\lambda}_{k}}, \eta\right]+\langle j k\rangle\left[\frac{\partial}{\partial \bar{\lambda}_{i}}, \eta\right]+\langle k i\rangle\left[\frac{\partial}{\partial \bar{\lambda}_{j}}, \eta\right] .
$$

The expressions for the NMHV gravity amplitudes can be used to test the twistor structure of gravity amplitudes. In this case, MHV amplitudes are annihilated by multiple applications of the collinearity operator,

$$
F^{h} M_{n}^{\mathrm{MHV}}=0,
$$

for some $h$. This is interpreted as the support being non-zero only if the points are "infinitesimally" close to a line in twistor space [5]. In ref. [36] it was explicitly shown that,

$$
\left[F_{i j k}, \eta\right]^{n-2} M_{n}^{\mathrm{MHV}}=0,
$$

for n-point amplitudes with $n \leq 8$. If we compare the action of the collinearity operator on the amplitude with that of the shift,

$$
\begin{aligned}
& \bar{\lambda}_{i} \rightarrow \bar{\lambda}_{i}+z\langle j k\rangle \bar{\eta}, \\
& \bar{\lambda}_{j} \rightarrow \bar{\lambda}_{j}+z\langle k i\rangle \bar{\eta}, \\
& \bar{\lambda}_{k} \rightarrow \bar{\lambda}_{k}+z\langle i j\rangle \bar{\eta},
\end{aligned}
$$

it can be seen that,

$$
\left[F_{i j k}, \eta\right] M_{n}(0)=\left.\frac{\partial}{\partial z} \hat{M}_{n}(z)\right|_{z=0}
$$


Equation (3.22) can thus be understood in terms of the number of $s_{i j}$ factors in the KLT form of the amplitude: each factor of $s_{i j}$ can introduce at most one power of $z$ and in the KLT form there are $n-3$ factors of $s_{i j}$ in the n-point amplitude, so $n-2$ applications of $\left[F_{i j k}, \eta\right]$ are sufficient to annihilate the amplitude.

\section{Beyond NMHV}

In this section we will illustrate a generalisation of the CSW-rules for gravity amplitudes with an example and verify the rules for this amplitude. Finally, we will present the generic rules and discuss their proof via the BCFW approach.

\subsection{General CSW rules}

We will now extend the CSW rules for NMHV amplitudes into more generic rules for the expansion of $\mathrm{N}^{n} \mathrm{MHV}$ amplitudes, that is amplitudes with $n+2$ negative helicity legs and the rest positive.

Consider the $\mathrm{N}^{n} \mathrm{MHV}$ amplitude with $N$ external legs. One would, as in the Yang-Mills case, begin by drawing all diagrams which may be constructed using MHV vertices. For the off-shell continuation, three-point MHV vertices are nonvanishing. The contribution from each diagram will be a product of $(n+1) \mathrm{MHV}$ vertices and $n$ propagators as indicated below.

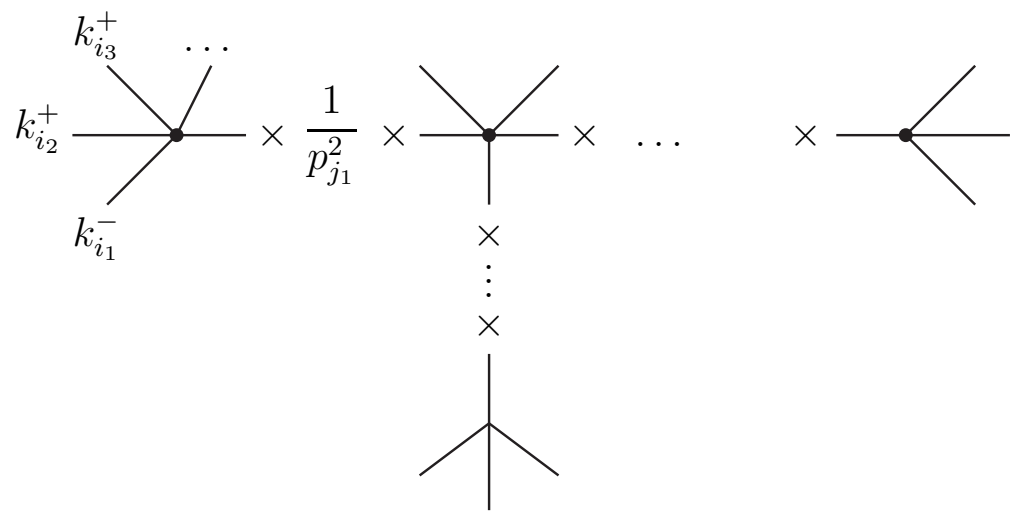

In contrast to gauge theory the CSW diagrams for gravity have no cyclic ordering of the external legs.

We denote internal momenta by $p_{j}$ for $j=1, \ldots, n$ and external momenta by $k_{i}$ for $i=1, \ldots, N$. We label the vertices by $l$ for $l=1, \ldots,(n+1)$. The momenta leaving MHV vertex $l$ are collected into the set $K_{l}$ and the number of external legs of MHV vertex $l$ will be denoted by $N_{l}$.

The contribution of a given diagram to the total amplitude can be calculated by evaluating the product of MHV amplitudes and propagators,

$$
\left.M_{N}^{n}\right|_{\mathrm{CSW} \text {-diagram }}=\left(\prod_{l=1, n+1} M_{N_{l}}^{\mathrm{MHV}}\left(\hat{K}_{l}\right)\right) \prod_{j=1, n} \frac{i}{p_{j}^{2}},
$$


where the propagators are computed on the set of momenta $k_{i}$ and $p_{j}$, and the MHV vertices are evaluated for the momenta $\hat{k}_{i}$ and $\hat{p}_{j}$. The definitions of these momenta are given below.

The momenta $k_{i}$ are the given external momenta and the internal momenta, $p_{j}$, are given by momentum conservation on each MHV-vertex.

The momenta $\hat{k}_{i}$ and $\hat{p}_{j}$ are uniquely specified so that they are massless and obey momentum conservation constraints at each vertex. Explicitly they are given by shifting the negative helicity legs $k_{i^{-}}$,

$$
\hat{k}_{i^{-}}=k_{i^{-}}+a_{i^{-}} \lambda_{\left(i^{-}\right)} \bar{\eta}
$$

and leaving the positive helicity legs $k_{i^{+}}$untouched. This introduces $n+2$ parameters, $a_{i^{-}}$. Overall momentum conservation is used to fix two of these parameters. Momentum conservation at each vertex then gives the momenta $\hat{p}_{j}$ as functions of $k_{i}$ and $a_{i}$. Finally the remaining parameters are fixed such that all internal momenta, $\hat{p}_{j}$, are massless,

$$
\hat{p}_{j}^{2}=0
$$

This gives $n$ further linear constraints which are sufficient to fix the remaining $a_{i}$ uniquely for a given spinor $\mid \eta]$. The MHV vertices in (4.1) can then be evaluated on the on-shell momenta $\hat{k}$ and $\hat{p}$.

\subsection{Example}

As an example we will discuss an explicit CSW diagram that contributes to the 8-point amplitude $M\left(1^{-}, 2^{-}, 3^{+}, 4^{+}, 6^{+}, 7^{-}, 8^{-}\right)$. The diagram is given by,

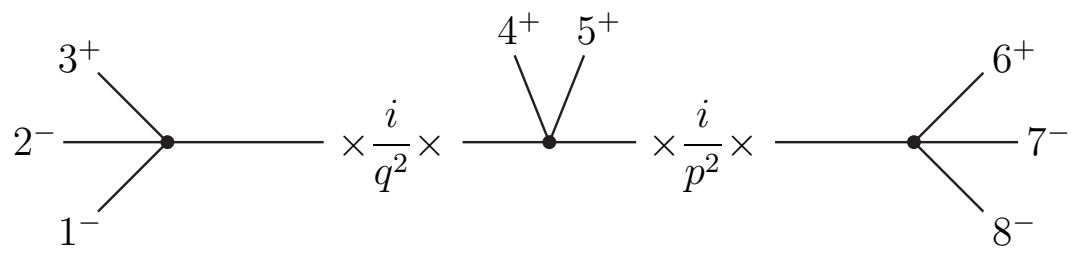

This specific diagram is interesting as none of its vertices can be written purely in terms of angles, \langle\rangle .

Following the algorithm from above the diagram contributes,

$$
\begin{aligned}
& M\left(\hat{1}^{-}, \hat{2}^{-}, 3^{+}, \hat{q}^{+}\right) \frac{i}{q^{2}} M\left((-\hat{q})^{-}, 4^{+}, 5^{+},(-\hat{p})^{-}\right) \frac{i}{p^{2}} M\left(\hat{p}^{+}, 6^{+}, \hat{7}^{-}, \hat{8}^{-}\right)= \\
& \frac{i\langle\hat{1} \hat{2}\rangle^{6}[3 \hat{q}]}{\langle\hat{2} 3\rangle\langle 3 \hat{q}\rangle\langle\hat{q} \hat{1}\rangle\langle\hat{2} \hat{q}\rangle\langle 3 \hat{1}\rangle} \frac{i}{q^{2}} \frac{i\langle\hat{p} \hat{q}\rangle^{6}[45]}{\langle\hat{q} 4\rangle\langle 45\rangle\langle 5 \hat{p}\rangle\langle\hat{q} 5\rangle\langle 4 \hat{p}\rangle} \frac{i}{p^{2}} \frac{i\langle\hat{7} \hat{8}\rangle^{6}[\hat{p} 6]}{\langle\hat{p} 6\rangle\langle 6 \hat{7}\rangle\langle\hat{8} 6\rangle\langle\hat{p} \hat{7}\rangle} .
\end{aligned}
$$

The internal momenta, $q$ and $p$, are given by momentum conservation: $q+k_{1}+k_{2}+$ $k_{3}=0$ and $p+k_{6}+k_{7}+k_{8}=0$. 
For the momenta $\hat{k}_{i}, i=1,2,7,8$, the shift is,

$$
\hat{k}_{i}=k_{i}+a_{i} \lambda_{i} \bar{\eta}, \quad i=1,2,7,8,
$$

while the momenta $k_{i}$ with $i=3,4,5,6$ are untouched. The momenta $\hat{p}$ and $\hat{q}$ and the parameters $a_{i}$ have to be fixed such that the momentum flowing through each of the vertices is preserved,

$$
\begin{aligned}
\sum_{i=1,8} \hat{k}_{i} & =0, \\
\hat{q}+\hat{k}_{1}+\hat{k}_{2}+k_{3} & =0, \\
\hat{p}+k_{6}+\hat{k}_{7}+\hat{k}_{8} & =0 .
\end{aligned}
$$

This leaves two free parameters which are fixed such that,

$$
\hat{q}^{2}=0, \quad \hat{p}^{2}=0
$$

There is a specific shift for each CSW diagram. In general, different diagrams that contribute to the same amplitude yield different values of $a_{i}$.

The various spinor products in (4.4) can be computed using the above conditions for the $a_{i}$ and one finds expressions very reminiscent of those derived by CSW for gauge theory,

$$
\begin{aligned}
& \left\langle k_{i} \hat{q}\right\rangle=\left\langle k_{i}\left|\left(-P_{123}\right)\right| \eta\right] / \omega_{q}, \quad \omega_{q}=[\hat{q} \eta], \\
& \left\langle k_{i} \hat{p}\right\rangle=\left\langle k_{i}\left|\left(-P_{678}\right)\right| \eta\right] / \omega_{p}, \quad \omega_{p}=[\hat{p} \eta] \\
& {[3 \hat{q}]=-\frac{\omega_{q}\left[3\left|\hat{P}_{123}\right| \hat{p}\right\rangle}{\left[\eta\left|\hat{P}_{123}\right| \hat{p}\right\rangle}=-\frac{\omega_{q}\left[3\left|\hat{P}_{123} \hat{P}_{678}\right| \eta\right]}{\left[\eta\left|\hat{P}_{123} \hat{P}_{678}\right| \eta\right]}=\frac{\omega_{q}\left[3\left|P_{45} P_{678}\right| \eta\right]}{\left[\eta\left|P_{123} P_{678}\right| \eta\right]},} \\
& {[6 \hat{p}]=-\frac{\omega_{p}\left[6\left|\hat{P}_{678}\right| \hat{q}\right\rangle}{\left[\eta\left|\hat{P}_{678}\right| \hat{q}\right\rangle}=-\frac{\omega_{p}\left[6\left|\hat{P}_{678} \hat{P}_{123}\right| \eta\right\rangle}{\left[\eta\left|\hat{P}_{678} \hat{P}_{123}\right| \eta\right\rangle}=\frac{\omega_{p}\left[6\left|P_{45} P_{123}\right| \eta\right]}{\left[\eta\left|P_{678} P_{123}\right| \eta\right]} .}
\end{aligned}
$$

Overall the $\omega_{q}$ and $\omega_{p}$ factors cancel and we find that (4.4) is given by,

$$
\begin{aligned}
& \frac{i\langle 12\rangle^{6} \frac{\left[3\left|P_{45} P_{678}\right| \eta\right]}{\left[\eta\left|P_{123} P_{678}\right| \eta\right]}}{\langle 23\rangle\left\langle 3\left|P_{123}\right| \eta\right]\left[\eta\left|P_{123}\right| 1\right\rangle\left\langle 2\left|P_{123}\right| \eta\right]\langle 31\rangle} \frac{i}{t_{123}} \frac{i\left[\eta\left|P_{678} P_{123}\right| \eta\right]^{6}[45]}{\left[\eta\left|P_{123}\right| 4\right\rangle\langle 45\rangle\left\langle 5\left|P_{678}\right| \eta\right]\left[\eta\left|P_{123}\right| 5\right\rangle\left\langle 4\left|P_{678}\right| \eta\right]} \\
& \times \frac{i}{t_{678}} \frac{i\langle 78\rangle^{6} \frac{\left[\eta\left|P_{123} P_{45}\right| 6\right]}{\left[\eta\left|P_{123} P_{678}\right| \eta\right]}}{\left\langle 8\left|P_{678}\right| \eta\right]\left[\eta\left|P_{678}\right| 6\right\rangle\langle 67\rangle\langle 86\rangle\left[\eta\left|P_{678}\right| 7\right\rangle} .
\end{aligned}
$$

The rules used to compute the above $\mathrm{N}^{2} \mathrm{MHV}$ diagram are a natural generalisation of the NMHV-case. As we will discuss below, they follow from BCFW recursions, providing the shifted amplitudes vanish for large $z$. 


\subsection{Proof of MHV-vertex rules}

In this section we prove the validity of the CSW-like expansion of the graviton scattering amplitudes in terms of MHV vertices with the substitution rules of the previous section. We will employ a recursive proof analogous to that for YangMills [22] where recursion was employed upon the number of minus legs in the tree amplitudes. As a first step we will prove the $\mathrm{N}^{2} \mathrm{MHV}$ case where four legs have negative helicity and later we will generalise the proof for generic $\mathrm{N}^{n} \mathrm{MHV}$ amplitudes.

\subsection{MHV-vertex Expansion for $\mathrm{N}^{2} \mathrm{MHV}$ Amplitudes}

We shall derive the CSW-like expansion for this amplitude by factorising the amplitude in two steps. First we shall factorise the amplitude into a product of MHV and NMHV amplitudes and then factorise the NMHV amplitudes to complete the expansion.

We first apply a holomorphic shift similar to the one discussed in [22] for gauge theory,

$$
\bar{\lambda}_{i} \longrightarrow \bar{\lambda}_{i}+z_{1} r_{i}^{(1)} \bar{\eta}, \quad i=1, \cdots, 4,
$$

where the $r_{i}^{(1)}$ are restricted by momentum conservation and are all non-zero. This shift of all negative helicity legs in $M^{\mathrm{N}^{2} \mathrm{MHV}}$ allows us to factorise the full amplitude as,

$$
M^{\mathrm{N}^{2} \mathrm{MHV}}=\sum_{\alpha} M^{\mathrm{MHV}}\left(z_{1, \alpha}\right) \frac{i}{P_{\alpha}^{2}} M^{\mathrm{NMHV}}\left(z_{1, \alpha}\right) .
$$

The summation is over all the physical factorisations of the amplitude. In the above the individual tree amplitudes are evaluated at the shifted momentum values. In particular the trees depend upon the shifted, on-shell, momenta $\hat{P}_{\alpha,(1)}$. We consider a single term in the summation corresponding to a specific pole,

$$
D_{\alpha}=M^{\mathrm{MHV}}\left(z_{1, \alpha}\right) \frac{i}{P_{\alpha}^{2}} M^{\mathrm{NMHV}}\left(z_{1, \alpha}\right),
$$

and evaluate this by determining the poles in $D_{\alpha}\left(z_{2}\right)$ under the shift,

$$
\bar{\lambda}_{i} \longrightarrow \bar{\lambda}_{i}+z_{2} r_{i}^{(2)} \bar{\eta}, \quad i=1, \cdots, 4 .
$$

The $r_{i}^{(2)}$ are restricted to maintain momentum conservation and to leave the pole unshifted,

$$
P_{\alpha}^{2} \longrightarrow P_{\alpha}^{2},
$$

which corresponds to the constraint,

$$
0=\sum_{i} z_{2} r_{i}^{(2)}\left[\eta\left|P_{\alpha}\right| i\right\rangle
$$

where $i$ runs over the indices $(1, \ldots, 4)$ which lie in the set $\alpha$. This condition also implies that the internal legs remain on-shell. 
This gives three linear constraints on the four $z_{2} r_{i}^{(2)}$. The function $D_{\alpha}\left(z_{2}\right)$ is rational and, since the two tree amplitudes do not have simultaneous poles, has simple poles. The poles occur where $M^{\mathrm{NMHV}}$ factorises into pairs of MHV amplitudes and thus, assuming $D_{\alpha}\left(z_{2}\right)$ vanishes at infinity, we have,

$$
D_{\alpha}=\sum_{\beta} D_{\alpha, \beta}=\sum_{\beta} M^{\mathrm{MHV}}(z) \frac{i}{P_{\alpha}^{2}} \times\left(M^{\mathrm{MHV}}(z) \frac{i}{P_{\beta,(1)}^{2}} M^{\mathrm{MHV}}(z)\right) .
$$

where $z$ indicates the functional dependance upon the two shifts (4.10) and (4.13). Explicitly, all three MHV amplitudes are evaluated at the shifted points,

$$
\bar{\lambda}_{i} \rightarrow \bar{\lambda}_{i}+\left(z_{1, \alpha} r_{i}^{(1)}+z_{2, \beta} r_{i}^{(2)}\right) \bar{\eta} .
$$

In eq. (4.16) we have a shifted propagator $P_{\beta,(1)}^{2}$ rather than $P_{\beta}^{2}$ since we are factorising the shifted tree amplitude $M^{\mathrm{NMHV}}$. Hence this is not immediatelly an MHV diagram term. but is one contribution to the MHV diagram with unshifted propagators $i / P_{\alpha}^{2}$ and $i / P_{\beta}^{2}$. There is a second contribution to the same MHV diagram which arises from the term with a $P_{\beta}^{2}$ pole in the sum in eq. (4.11). Expansion of this yields,

$$
D_{\beta, \alpha}=\left(M^{\mathrm{MHV}}\left(z^{\prime}\right) \frac{i}{P_{\alpha,(1)}^{2}} M^{\mathrm{MHV}}\left(z^{\prime}\right)\right) \times \frac{i}{P_{\beta}^{2}} M^{\mathrm{MHV}}\left(z^{\prime}\right),
$$

where the MHV amplitudes are now evaluated at the points

$$
\bar{\lambda}_{i} \rightarrow \bar{\lambda}_{i}+\left(z_{1, \beta} r_{i}^{(1)}+z_{2, \alpha}^{\prime} r_{i}^{(2)}\right) \bar{\eta}
$$

To prove the MHV-diagram expansion we need to show that the sum of the two terms $D_{\alpha, \beta}+D_{\beta, \alpha}$ gives the correct diagram, i.e.,

$$
\begin{gathered}
M^{\mathrm{MHV}}(z) \frac{i}{P_{\alpha}^{2}}\left(M^{\mathrm{MHV}}(z) \times \frac{i}{P_{\beta,(1)}^{2}} M^{\mathrm{MHV}}(z)\right) \\
+\left(M^{\mathrm{MHV}}\left(z^{\prime}\right) \frac{i}{P_{\alpha,(1)}^{2}} M^{\mathrm{MHV}}\left(z^{\prime}\right)\right) \times \frac{i}{P_{\beta}^{2}} M^{\mathrm{MHV}}\left(z^{\prime}\right) \\
=M^{\mathrm{MHV}}\left(z_{a}\right) \frac{i}{P_{\alpha}^{2}} M^{\mathrm{MHV}}\left(z_{a}\right) \frac{i}{P_{\beta}^{2}} M^{\mathrm{MHV}}\left(z_{a}\right)
\end{gathered}
$$

with the $M^{\mathrm{MHV}}\left(z_{a}\right)$ evaluated at the point $z_{a}$ specified by the rules of the previous section.

We need two facts to show this:

- The product of the three tree amplitudes is the same in both cases and equal to the desired value. This is equivalent to showing that $z \equiv z^{\prime} \equiv z_{a}$.

- There is an identity involving the product of propagators,

$$
\frac{i}{P_{\alpha}^{2}} \frac{i}{P_{\beta,(1)}^{2}}+\frac{i}{P_{\alpha,(1)}^{2}} \frac{i}{P_{\beta}^{2}}=\frac{i}{P_{\alpha}^{2}} \frac{i}{P_{\beta}^{2}} .
$$


Taking the first fact: in the final expression for the $\bar{\lambda}_{i}$ the net effect of the two shifts is to give a total shift of the form,

$$
\hat{\bar{\lambda}}_{i}=\bar{\lambda}_{i}+a_{i} \bar{\eta}
$$

The $a_{i}$ are such that momentum conservation is satisfied and $\hat{P}_{\alpha,(2)}^{2}=\hat{P}_{\beta,(2)}^{2}=0$. As discussed in the previous section, these constraints have a unique solution and so the

$\bar{\lambda}_{i}$ take the same values irrespective of the order in which we factorise. The values of the intermediate momenta, $\hat{P}$, are determined by momentum conservation which are precisely the substitutions specified in the substitution rules.

The second fact can be shown in the following way. Considering the contour integral of two shifted propagators

$$
\oint \frac{d z}{z} \frac{1}{P_{\alpha}^{2}(z) P_{\beta}^{2}(z)}
$$

about a contour at infinity. Since the $P_{\alpha}^{2}(z)$ vanish at infinity this integral vanishes and is also equal to the sum of its residues. Examining the residues we obtain,

$$
\frac{1}{P_{\alpha}^{2}} \frac{1}{P_{\beta}^{2}}-\frac{1}{P_{\alpha}^{2}\left(z_{\beta}\right)} \frac{1}{P_{\beta}^{2}}-\frac{1}{P_{\alpha}^{2}} \frac{1}{P_{\beta}^{2}\left(z_{\alpha}\right)}=0,
$$

which provides a proof of eq.(4.21).

Thus the two terms combine to give a single term which is the MHV-vertex diagram.

\subsection{General Case}

The general case can be deduced by a repeated application of the process used in the previous section. We give an outline of this here. Consider a general $\mathrm{N}^{n} \mathrm{MHV}$ amplitude and shift all the negative helicity legs,

$$
\bar{\lambda}_{i} \rightarrow \bar{\lambda}_{i}+z_{1} r_{i}^{(1)} \bar{\eta}
$$

for a generic set of $r_{i}^{(1)}$. The amplitude can then be written as,

$$
M^{n}(0)=\sum_{\alpha} M^{n-k_{\alpha}+1}(\ldots, \hat{p})\left(z_{1, \alpha}\right) \frac{i}{P_{\alpha}^{2}} M^{k_{\alpha}+1}((-\hat{p}), \ldots)\left(z_{1, \alpha}\right) .
$$

We evaluate an individual term in this by imposing a shift with parameter $z_{2}$ that does not shift $P_{\alpha}^{2}$. We continue in this way until the we have an amplitude which is a product of MHV amplitudes with propagators,

$$
D_{\alpha_{1}, \alpha_{2}, \cdots \alpha_{n}}=\prod\left(M^{\mathrm{MHV}}\right) \times \prod_{i} \frac{i}{P_{\alpha_{i},(i-1)}^{2}},
$$


where $i / P_{\alpha_{i},(i-1)}^{2}$ denotes the propagator we factorised on in the $i$-th step. As before we gather together all terms with the same pole structure and combine them into a single diagram. This again requires two things: firstly that the MHV amplitudes are evaluated at the same point irrespective of the order and secondly that the pole terms sum to yield the product of the unshifted poles.

For the first step we note that the net effect of the shifts is to apply an overall shift to the $n+2$ negative helicity legs of the form,

$$
\hat{\bar{\lambda}}_{i}=\bar{\lambda}_{i}+a_{i} \bar{\eta} .
$$

Since momentum conservation is preserved at each step, overall momentum conservation is guaranteed at the final stage. This is equivalent to two linear constraints on the $a_{i}$. Secondly, the net effect at their final stage is that all the $\hat{P}_{\alpha_{i}}$ are on-shell, $\hat{P}_{\alpha_{i}}^{2}=0$. This imposes $n$ further linear constraints and we are left with a unique shift.

Summing over the different orderings now gives an expression of the form,

$$
\left.M^{n}(0)\right|_{\mathrm{CSW} \text {-diagram }}=\left(\prod M^{\mathrm{MHV}}\right)\left(\sum_{\sigma} \prod_{i} \frac{i}{\hat{P}_{\alpha_{\sigma(i)},(i-1)}^{2}}\right),
$$

where $\sigma$ denote the permutations of the labels $i=1, \ldots, n$. The rather complicated sum in (4.29) simply yields the product of propagators, as can be seen by comparing with the Yang-Mills case.

As the total amplitude can be expressed as a sum of terms, each with a specific pole structure, the $\mathrm{N}^{n} \mathrm{MHV}$ amplitude $M^{n}(0)$ can be written in a CSW form,

$$
M^{n}(0)=\left.\sum_{\text {CSW-diagram }} M^{n}(0)\right|_{\text {CSW-diagram }},
$$

with each CSW diagram contributing as

$$
\left.M^{n}(0)\right|_{\mathrm{CSW} \text {-diagram }}=\left(\prod M^{\mathrm{MHV}}\right) \prod_{i} \frac{i}{P_{\alpha_{i}}^{2}},
$$

as given in the rules of the previous section.

\section{Conclusions and Comments}

In this paper we have shown a new way of obtaining amplitudes for graviton scattering, using a gravity MHV-vertex formalism that resembles the CSW formalism for calculating tree amplitudes in Yang-Mills theory. Given the assumption that gravity amplitudes are sufficiently well behaved under a BCFW-style analytic continuation to complex momenta, we have presented a direct proof of the formalism 
and have illustrated its usefulness through concrete examples such as NMHV amplitudes. Although we have presented MHV-vertices for external gravitons only we expect the procedure to extend to other matter types using supersymmetry to obtain the relevant MHV-vertex [6,39].

Although the existence of the CSW formalism can be motivated by the duality with a twistor string theory, such a motivation is not so clear for gravity. The natural candidate string theories contain conformal supergravity [40] rather than conventional gravity. Despite this conventional gravity does seem to share features with Yang-Mills theory such as the existence of a MHV-vertex construction and the coplanarity [36] of NMHV amplitudes which hint at the existence of a twistor string dual theory.

\section{Acknowledgments}

We thank Zvi Bern for many useful discussions. This research was supported in part by the PPARC and the EPSRC of the UK. 


\section{References}

[1] F. A. Berends and W. Giele, Nucl. Phys. B 294, 700 (1987);

M. L. Mangano, S. J. Parke and Z. Xu, Nucl. Phys. B 298, 653 (1988);

M. L. Mangano, Nucl. Phys. B 309, 461 (1988).

[2] S. J. Parke and T. R. Taylor, Phys. Rev. Lett. 56, 2459 (1986);

F. A. Berends and W. T. Giele, Nucl. Phys. B 306, 759 (1988).

[3] Z. Xu, D. H. Zhang and L. Chang, Nucl. Phys. B 291, 392 (1987).

[4] M. Mangano and S.J. Parke, Phys. Rep. 200:301 (1991).

[5] E. Witten, Commun. Math. Phys. 252, 189 (2004) [hep-th/0312171].

[6] V. P. Nair, Phys. Lett. B 214, 215 (1988).

[7] F. Cachazo, P. Svrček and E. Witten, JHEP 0409, 006 (2004)

[hep-th/0403047].

[8] R. Roiban, M. Spradlin and A. Volovich, JHEP 0404, 012 (2004)

[hep-th/0402016];

C. J. Zhu, JHEP 0404, 032 (2004) [hep-th/0403115];

R. Roiban, M. Spradlin and A. Volovich, Phys. Rev. D 70, 026009 (2004)

[hep-th/0403190];

I. Bena, Z. Bern and D. A. Kosower, Phys. Rev. D 71, 045008 (2005)

[hep-th/0406133];

D. A. Kosower, Phys. Rev. D 71, 045007 (2005) [hep-th/0406175];

G. Georgiou, E. W. N. Glover and V. V. Khoze, JHEP 0407, 048 (2004)

JHEP 0407, 048 (2004) [hep-th/0407027];

R. Roiban, M. Spradlin and A. Volovich, Phys. Rev. Lett. 94, 102002 (2005) [hep-th/0412265].

[9] F. Cachazo, P. Svrček and E. Witten, JHEP 0410, 077 (2004)

[hep-th/0409245];

I. Bena, Z. Bern, D. A. Kosower and R. Roiban, Phys. Rev. D 71, 106010 (2005) [hep-th/0410054];

R. Britto, F. Cachazo and B. Feng, Phys. Rev. D 71, 025012 (2005)

[hep-th/0410179];

Z. Bern, V. Del Duca, L. J. Dixon and D. A. Kosower, Phys. Rev. D 71, 045006 (2005) [hep-th/0410224];

S. J. Bidder, N. E. J. Bjerrum-Bohr, L. J. Dixon and D. C. Dunbar, Phys. Lett. B 606, 189 (2005) [hep-th/0410296]; 
S. J. Bidder, N. E. J. Bjerrum-Bohr, D. C. Dunbar and W. B. Perkins, Phys. Lett. B 608, 151 (2005) [hep-th/0412023];

R. Britto, F. Cachazo and B. Feng, hep-th/0412103;

J. Bedford, A. Brandhuber, B. Spence and G. Travaglini, Nucl. Phys. B 712, 59 (2005) [hep-th/0412108];

Z. Bern, L. J. Dixon and D. A. Kosower, Phys. Rev. D 72, 045014 (2005)

[hep-th/0412210];

S. J. Bidder, N. E. J. Bjerrum-Bohr, D. C. Dunbar and W. B. Perkins, Phys. Lett. B 612, 75 (2005) [hep-th/0502028];

R. Britto, E. Buchbinder, F. Cachazo and B. Feng, hep-ph/0503132;

S. J. Bidder, D. C. Dunbar and W. B. Perkins, hep-th/0505249.

[10] G. Georgiou and V. V. Khoze, JHEP 0405, 070 (2004) [hep-th/0404072];

J. B. Wu and C. J. Zhu, JHEP 0409 (2004) 063 [hep-th/0406146];

X. Su and J. B. Wu, Mod. Phys. Lett. A 20, 1065 (2005) [hep-th/0409228];

L. J. Dixon, E. W. N. Glover and V. V. Khoze, JHEP 0412, 015 (2004) [hep-th/0411092].

[11] L. J. Dixon, E. W. N. Glover and V. V. Khoze, JHEP 0412, 015 (2004) [hep-th/0411092];

Z. Bern, D. Forde, D. A. Kosower and P. Mastrolia, Phys. Rev. D 72, 025006 (2005) [hep-ph/0412167];

S. D. Badger, E. W. N. Glover and V. V. Khoze, JHEP 0503, 023 (2005)

[hep-th/0412275]. S. D. Badger, E. W. N. Glover, V. V. Khoze and P. Svrček, JHEP 0507, 025 (2005) [hep-th/0504159];

D. Forde and D. A. Kosower, hep-th/0507292.

[12] A. Brandhuber, B. Spence and G. Travaglini, Nucl. Phys. B 706, 150 (2005) [hep-th/0407214].

[13] C. Quigley and M. Rozali, JHEP 0501, 053 (2005) [hep-th/0410278].

[14] J. Bedford, A. Brandhuber, B. Spence and G. Travaglini, Nucl. Phys. B 706, 100 (2005) [hep-th/0410280].

[15] Z. Bern, L. J. Dixon, D. C. Dunbar and D. A. Kosower, Nucl. Phys. B 425, 217 (1994) [hep-ph/9403226].

[16] Z. Bern, L. J. Dixon, D. C. Dunbar and D. A. Kosower, Nucl. Phys. B 435, 59 (1995) [hep-ph/9409265].

[17] R. Britto, F. Cachazo and B. Feng, Nucl. Phys. B 715, 499 (2005) [hep-th/0412308]; 
R. Britto, F. Cachazo, B. Feng and E. Witten, Phys. Rev. Lett. 94, 181602 (2005) [hep-th/0501052].

[18] M. Luo and C. Wen, JHEP 0503, 004 (2005), [hep-th/0501121];

[hep-th/0502009].

[19] R. Britto, B. Feng, R. Roiban, M. Spradlin and A. Volovich, Phys. Rev. D 71, 105017 (2005) [hep-th/0503198].

[20] Z. Bern, L. J. Dixon and D. A. Kosower, Phys. Rev. D 71, 105013 (2005)

[hep-th/0501240];

Z. Bern, L. J. Dixon and D. A. Kosower, hep-ph/0505055;

Z. Bern, L. J. Dixon and D. A. Kosower, hep-ph/0507005.

[21] Zvi Bern, N. E. J. Bjerrum-Bohr, David C. Dunbar and Harald Ita, hep-ph/0507019.

[22] K. Risager, hep-th/0508206

[23] F. A. Berends, W. T. Giele and H. Kuijf, Phys. Lett. B 211, 91 (1988).

[24] J. Bedford, A. Brandhuber, B. Spence and G. Travaglini, Nucl. Phys. B 721, 98 (2005) [hep-th/0502146].

[25] F. Cachazo and P. Svrček, hep-th/0502160.

[26] N. E. J. Bjerrum-Bohr, D. C. Dunbar and H. Ita, Phys. Lett. B 621, 183 (2005) [hep-th/0503102].

[27] H. Kawai, D. C. Lewellen and S. H. H. Tye, Nucl. Phys. B 269, 1 (1986).

[28] Z. Bern and D. A. Kosower, Phys. Rev. Lett. 66, 1669 (1991);

Nucl. Phys. B 379, 451 (1992);

Z. Bern, Phys. Lett. B 296, 85 (1992);

Z. Bern, D. C. Dunbar and T. Shimada, Phys. Lett. B 312, 277 (1993) [hep-th/9307001];

Z. Bern and D. C. Dunbar, Nucl. Phys. B 379, 562 (1992).

[29] Z. Bern, L. J. Dixon, M. Perelstein and J. S. Rozowsky, Phys. Lett. B 444, 273 (1998) [hep-th/9809160].

[30] Z. Bern, A. De Freitas and H. L. Wong, Phys. Rev. Lett. 84, 3531 (2000) [hep-th/9912033].

[31] Z. Bern, Living Rev. Rel. 5, 5 (2002) [gr-qc/0206071]. 
[32] N. E. J. Bjerrum-Bohr, Phys. Lett. B 560, 98 (2003) [hep-th/0302131]; Nucl. Phys. B 673, 41 (2003) [hep-th/0305062];

N. E. J. Bjerrum-Bohr and K. Risager, Phys. Rev. D 70, 086011 (2004) [hep-th/0407085].

[33] Z. Bern, L. J. Dixon, D. C. Dunbar, M. Perelstein and J. S. Rozowsky, Nucl. Phys. B 530, 401 (1998) [hep-th/9802162]; Class. Quant. Grav. 17, 979 (2000) [hep-th/9911194].

[34] Z. Bern, L. J. Dixon, M. Perelstein and J. S. Rozowsky, Nucl. Phys. B 546, 423 (1999) [hep-th/9811140].

[35] D. C. Dunbar and P. S. Norridge, Nucl. Phys. B 433, 181 (1995) [hep-th/9408014];

D. C. Dunbar and N. W. P. Turner, Class. Quant. Grav. 20, 2293 (2003) [hep-th/0212160];

D. C. Dunbar, B. Julia, D. Seminara and M. Trigiante, JHEP 0001, 046 (2000) [hep-th/9911158].

[36] Z. Bern, N. E. J. Bjerrum-Bohr and D. C. Dunbar, JHEP 0505, 056 (2005) [hep-th/0501137].

[37] S. Giombi, R. Ricci, D. Robles-Llana and D. Trancanelli, JHEP 0407, 059 (2004) [hep-th/0405086].

[38] J. B. Wu and C. J. Zhu, JHEP 0407, 032 (2004) [hep-th/0406085].

[39] V.P. Nair, hep-th/0501143

[40] N. Berkovits and E. Witten, JHEP 0408 (2004) 009 [hep-th/0406051];

Phys. Rev. Lett. 93, 011601 (2004) [hep-th/0402045]. 